\title{
Research progress on the vesicle cycle and neurological disorders
}

\author{
Li-Juan Zhu, Cheng-Cheng Zhang, Ce Chen
}

Key Laboratory of Developmental Genes and Human Diseases, Ministry of Education, Department of Histology and Embryology, School of Medicine, Southeast University, Nanjing, Jiangsu 210009, PR China.

Corresponding author: Li-Juan Zhu, Key Laboratory of Developmental Genes and Human Diseases, Department of Histology and Embryology, School of Medicine, Southeast University, Nanjing, Jiangsu 210009, PR China. email: zhulj@seu.edu.cn

Received, June 10, 2021; Revised, July 7, 2021; Accepted, July 25, 2021; Published, July 30, 2021

\begin{abstract}
Neurons are special polarized cells whose synaptic vesicles release neurotransmitters into the synaptic cleft, acting on postsynaptic receptors and thus transmitting information from presynaptic to postsynaptic states. The integrity of the vesicle cycle is critical to the transmission of neural signals in the brain. According to the molecular mechanism of calcium-triggered release, the assembly of soluble $\mathrm{N}$ ethylmaleimide-sensitive factor attachment protein receptors (SNAREs) is required in the process of synaptic vesicle fusion and vesicle exocytosis. Many delicate steps are required to maintain the dynamic process of 'release-recycle', including intermediate processes and the dynamic balance of neurotransmission. Various neurodegenerative and neuropsychiatric diseases result from synaptic cycle dysfunction. This review of the relationships between the structure and function of synaptic vesicles in physiological and pathological conditions provides a theoretical basis for synaptic transmission and a novel avenue for the study of synaptic plasticity associated with mood disorders, highlighting potential targets for treating diseases.

Abbreviations. SNARE: soluble N-ethylmaleimide-sensitive factor attachment protein receptors; RRP: readily releasable pool; LRRK2: leucine-rich repeat kinase 2; CDK5: cyclin-dependent kinase 5; SNAP: synaptosome-associated protein; VAMP: vesicle-associated membrane protein; CME: clathrin-mediated endocytosis; ADBE: activity-dependent bulk endocytosis; a-Syn: a-synuclein; PD: Parkinson's Disease; DAergic: dopaminergic; SN: substantia nigra; SV: synaptic vesicle; A $\beta$ : amyloid- $\beta$; APP: amyloid precursor protein; HD: Huntington's disease; HTT: huntingtin protein; WHO: World Health Organization; NSF: neurexin/N-ethylmaleimide sensitive factor; NMDARs: NMDA receptors
\end{abstract}

\section{VESICLE POOLS AND THE SYNAPTIC CYCLE}

Greengard's laboratory (1) found that synaptic vesicles bound with synapsin initiate migration to the presynaptic membrane under low-frequency stimulation, while other vesicles migrate to the presynaptic membrane only under high-frequency stimulation, indicating that synapsin, as a chaperone, facilitates the fusion of vesicles to the presynaptic membrane and the release of neurotransmitters. Moreover, numerous reports have identified that synaptic vesicles can be segmented into class 'pools' according to function and morphology, including the readily releasable pool (docked in the active zone near the presynaptic membrane and ready to release), the recycling pool (recycled upon moderate stimulation and supplied with the readily releasable pool under physiological stimulation) and the reserve pool (occupying most of the vesicle clusters and released upon non-physiological stimulation) (2). As the classification of vesicles is a complicated research process, several new research findings have optimized the three-pool model and proposed new concepts for spontaneously recycling the vesicle pool (3).

The readily releasable pool (RRP) refers to a group of synaptic vesicle anchors on the presynaptic membrane that initiate release immediately upon stimulation, which determines the sensitivity of synaptic cycling (4). With the depletion of RRP vesicles, the intensity of synaptic contact gradually decreases, though it is extremely sensitive to stimulation at the beginning (5). Whether RRPreleased vesicles can be quickly recovered, that is, whether the recovered vesicles can be directly returned to RRP, is a controversial issue that was raised in 2007 (6). Tsien et al. (7) found that recovered vesicles could be re-released quickly with FM dye, indicating that RRP vesicles could be quickly reused. However, Murthy's lab (8) found that recovered vesicles entered the reserve pool rather than the readily releasable pool (RRP).

The recycling pool is a part of vesicles that can be continuously circulated under physiological stimulation and are utilized to replenish the RRP. Research has shown that the recycling pool plays a decisive role in maintaining release under continuous stimulation, while the stage of the recycling pool is always in the process of dynamic change. Therefore, the replenishment of the recycling pool to the RRP is important for the maintenance of the synaptic cycle (9). Meanwhile, it has been reported that the storage and mobilization of the recycling pool are controlled by leucine-rich repeat kinase 2 (LRRK2), including vesicle 
trafficking and distribution in neurons (10). Moreover, recent studies have shown that the rate of RRP supplementation will be accelerated with increasing neuron activity and temperature (11). In addition, the rate of RRP supplementation is also accelerated by the activation of protein kinase $\mathrm{C}$ (12). Interestingly, a study found that the antidepressant fluoxetine enhances the recycling vesicle pool size in cultured rat hippocampal neurons $(13,14)$.

The reserve pool refers to the storage of vesicles released under high-intensity stimulation. It occupies most of the vesicle clusters, and its range is from $50 \%$ to $85 \%$ according to different experimental tests (15). The mobilization of RP vesicles can be regulated by various molecules in vivo $(16,17)$. For example, it has been shown that CDK5, located in the synaptic terminus of hippocampal neurons, possesses negative effects on the mobilization of the reserve pool (18). As a supplementary reserve, the reserve pool also supports neurotransmission indirectly and ensures the delivery of soluble recycling proteins in synaptic activity (9).

Under the regulation of a large number of molecular chaperones, neurotransmitters in synaptic vesicles transfer from the presynaptic membrane to the postsynaptic membrane receptor and cause the depolarization of the postsynaptic membrane, which ensures the neurotransmission cycle from electrical signals to chemical signals and then to electrical signals. Thus, it seems that the disruption of the synaptic vesicle cycle may lead to neurological disorders.

\section{Process of the vesicle cycle}

Exocytosis is more complex than one might think. The origin of synaptic vesicles between the Golgi complex and endoplasmic reticulum has been continuously investigated. Soluble Nethylmaleimide-sensitive factor attachment protein receptors (SNAREs) are required in synaptic vesicle fusion and vesicle exocytosis $(19,20)$. In most cases, the process of the vesicle cycle can be summarized as the docking of vesicles containing neurotransmitters in the active zone near the presynaptic membrane in preparation for action potential attainment and endocytosis $(21,22)$. Actin, transporters, and other proteins recruited around the vesicles are generated freshly in an energydependent manner and are allowed to be transported to vesicle pools as needed. Before the voltagesensitive calcium channel is opened, syntaxin-1 is in a closed state induced by Munc18 of the SM family, which combines with the $\mathrm{N}$-terminal sequence and Habc domain of syntaxin-1(23). With the MUN domain of Munc13 interacting with syntaxin-1(24), the complex conformation of Munc-18 and syntaxin1 transforms from closed to open and exposes the SNARE binding site, then combines with synaptosome-associated protein (SNAP-25) on the plasma membrane and vesicle-associated membrane protein (VAMP) distributed on synaptic vesicles, and then assembles into a tight trans-SNARE complex at a ratio of $1: 1: 1(25-28)$. The vesicle membrane is forced to bind tightly to the presynaptic membrane by coiled helices in the core region and gradually compresses from the $\mathrm{N}$-terminus to the $\mathrm{C}$-terminus, resulting in the loss of hydrophilic surface stability $(29,30)$. Then, vesicles in the pre-fusion stage with the trans-SNARE complex attach to the coiled helical domain. As the action potential transmitted to the nerve terminal and the voltage-sensitive calcium ion channels opened, vesicle fusion initiated rapid release, in which synaptotagmin-1 binds to calcium ions through the $\mathrm{C} 2$ domain $(31,32)$. With the $\mathrm{C} 2$ domain of synaptotagmin-1 allosteric after binding with calcium ions, the clamping effect on the SNARE complex is eliminated. Inserting the Cterminus of synaptotagmin-1 into the presynaptic membrane increases the local curvature of the presynaptic membrane and significantly reduces the energy barrier of the fusion pore. When vesicles fuse with the presynaptic membrane to form fusion holes, neurotransmitters diffuse into the synaptic clefts and bind to relevant receptors on the postsynaptic membrane, triggering transmembrane ion flow and generating a change in the postsynaptic membrane potential, which transmits the signals $(33,34)$. Fusion pore expansion converts the initial transSNARE complex into a cis-SNARE complex, where SNAP and NSF are directly recruited into the SNARE complex. With ATPase, the SNARE complexes dissociate into free monomer proteins to participate in a new round of exocytosis. (35).

Studies have shown that a large number of proteins are involved in the regulation of neurotransmitter release. For example, in addition to forming a complex with Munc18, syntaxin-1 addresses a unique functional form by interacting with SNAP25 and VAMP2 in trans-SNARE complex assembly with Munc13 and shows interactions with synaptotagmin- 1 , synaphin and calcium ion channels Through the identification of the mechanism of vesicle exocytosis, a model has been proposed in which Munc18 combines with the N-terminus of syntaxin-1 to participate in the process of SNARE complex assembly and the assembly of the SNARE complex regulated by synaphin and synaptotagmin1. Moreover, exposing the supportive role of the depolymerized syntaxin-1-SNAP25 heterodimer, evidence has shown that NSF and SNAP act as chaperones that facilitate the disassembly of the cisSNARE complex during the SNARE cycle, which may be related to the maintenance of the syntaxin-1munc18 complex. Obviously, any errors in the process of neurotransmitter release mediated by the SNARE complex may disrupt the cycle of vesicle exocytosis, as well as the synaptic transmission of fidelity and plasticity, resulting in neurodegenerative and neuropsychiatric disorders. 


\section{MODES OF SV RECYCLING}

At present, more than four SV recycling modes have been described, which are classified according to their molecular mechanisms and speeds: clathrinmediated endocytosis, kiss-and-run, ultrafast endocytosis, and activity-dependent bulk endocytosis.

\section{Clathrin-mediated endocytosis}

Conceptually, clathrin-mediated endocytosis (CME) is a fairly simple process in which small membrane vesicles are generated, each containing various cargo molecules that are then transported from the cell surface to the interior of eukaryotic cells (36). As the major endocytic pathway for internalization of many cargoes, CME is a classic approach for the recycling of synaptic vesicles. The physiological processes of CME were explained over 5 decades ago, including clathrin coats, coated pit maturation, fission and uncoating. This process is initiated after the full fusion of synaptic vesicles, which can be recognized by adaptor 2, and endocytic coat proteins are recruited from the cytosol to the inner leaflet of the plasma membrane $(37,38)$.

A double layer of clathrin packets usually consists of external clathrin and inner adaptors. Adaptors cooperate with clathrin protein to promote membrane coating (37). The flat plasma membrane is transformed into a 'clathrin-coated pit' by curvature proteins directly or indirectly connected to the lipid bilayer. In vitro experiments revealed that clathrin and other auxilin are involved in the formation of these pits, but this cannot be accomplished by clathrin alone. Then, clathrincoated vesicles are produced from the plasma membrane after the neck of the membrane is ruptured and are powered by dynamin protein as a GTPase. After the destruction of dynamin expression, synaptic transmission is inhibited, and vesicles remain immature, although they continue to fuse with the plasma membrane, consistent with the dysfunction of vesicle endocytosis in dynaminmutant mice. Finally, the endocytic protein machinery disassembles quickly, releasing nascent vesicles and allowing them to track into the nest vesicle to circulate. More details about CME can be found in recent articles (39).

CME is a protein that plays a critical role in the endocytic machinery. To date, over 50 other cytosolic proteins have been identified to be involved in the process of CME (40). All of these proteins have been examined in detail by biochemical and biophysical methods, and their activities have been shown to facilitate different steps of endocytosis. However, understanding how these proteins work together in a highly coordinated manner in vesicle endocytosis is still a major focus of study.

\section{Kiss-and-run}

The kiss-and-run mechanism was first discovered in 1994 in an experiment involving the frog neuromuscular junction (41). In contrast to the classical CME mechanism, kiss-and-run is faster than CME, occurring in 2 seconds, while CME is relatively slow $(10-30 \mathrm{~s})$ and contains a distinct set of molecules recruited from the cytosolic pool to the interior. With the opening of the transient instantaneous nanoscale fusion channel and the release of neurotransmitters, the cargoes are recruited into synaptic vesicles in the active zone and are further transported to the next vesicle cycle (42, $43)$. Nonetheless, the essential role of kiss-and-run in the release of neurotransmitters from synaptic vesicles has been questioned. This hypothesis has long been controversial; that is, kiss-and-run has been considered a compensation pathway for vesicle endocytosis. Since kiss-and-run and CME coexist during the process of neurotransmitter release in the central nervous system, the function of kiss-and-run still needs to be further evaluated.

Moreover, assembly of the SNARE complex is required for kiss-and-run activity after intense activity, and the SNARE complex works in conjunction with other factors in the cell membrane during instantaneous fusion pore formation (44). A unified view from these studies has led to the idea that actin cooperates with the SNARE complex to promote cargo trafficking from synaptic vesicles in a kiss-and-run manner. Using a palette of smallmolecule dynamin modulators, the critical role of dynamin presented in the exocytosis fusion pore and vesicle release were determined to function as bidirectional regulators (45), while the endocytosis of vesicles were blocked in dynamin mutation mice in the kiss-and-run pathway.

\section{Ultrafast endocytosis}

Compared to kiss-and-run endocytosis, ultrafast endocytosis can complete in as fast as $50 \mathrm{~ms}$ (46). Ultrafast endocytosis is trafficked immediately to endosomes and regenerates new SVs in a clathrinindependent manner. This mode of SV recycling occurs at the lateral edges of an active zone in C. elegans NMJs and mouse central synapses (47). It operates after a sparse level of neuronal activity, but it may also occur during high-frequency stimulation in mammalian cells (48). Since ultrafast endocytosis was first observed in cultured hippocampal neurons with a single stimulus (49), it is worth a more thorough investigation because it is as important as other modes of SV recycling of the molecular mechanisms, and it is a form of clathrin-independent endocytosis.

Additionally, to maintain unremitting neurotransmission, 'ultrafast endocytosis' has been attributed to the thinking that the major endocytic pathway occurs within 50 to $100 \mathrm{~ms}$, which is 
characterized by the rapid cycle between the active zone and endocytic sites mediated by dynamin and other relaxation proteins (50). Ultrafast endocytosis has also been observed to share many molecular participants with other endocytic pathways, including kiss-and-run. For example, synaptojanin and endophilin, two key auxilins in kiss-and-run, coordinate scissoring the narrow neck between invaginated budding vesicle and plasma membrane, while the endocytosis fails completely in the presence of inhibitors $(51,52)$. Ultrafast endocytosis has only recently been discovered; the exact mechanism remains to be elucidated.

\section{Activity-dependent bulk endocytosis}

Another mode for SV recycling is called activitydependent bulk endocytosis (ADBE). Under highfrequency stimulation, $\mathrm{ADBE}$ is the dominant mode of SV endocytosis, while CME, kiss-and-run and ultrafast endocytosis occur with stimulation at low activity (53). ADBE is triggered by longer bursts of intense activity with a large flat plasma membrane that has been invaginated to form an intracellular endosome within 1-2 s in a clathrin-independent process. This rigorous procedure of ADBE can be influenced by the activation of phosphatase calcineurin in a calcium-dependent manner (54). Moreover, several studies have reported that the efflux of previously accumulated extracellular calcium promotes the budding of SV from intracellular endosomes, which is driven by endosomal acidification $(55,56)$. Interestingly, the important role of the actin cytoskeleton in ADBE has been presented, suggesting that the formation of bulk endosomes may be coupled to neuronal activity (57). Although the physiological mechanism of ADBE is unclear, recent studies have indicated that ADBE can recycle typical SV cargoes such as VAMP2 and synaptophysin, while VAMP4 preferentially accumulates by ADBE and plays a vital role in endocytosis $(58,59)$. Consequently, AP-2 plays a major role in the cargo sorting process due to the shallower membrane curvature of the larger bulk endosomes, whereas AP-1/AP-3 may be involved in smaller endosomes, indicating different adaptor proteins assemble in fresh SVs (60).

\section{SYNAPTIC CYCLE IMPAIRMENT AND NEUROPSYCHIATRIC DISEASES}

Neurotransmitters are released from synaptic vesicles in presynaptic terminals. As the intermediate of reactive SNARE proteins, the assembly and disassembly cycles of SNARE complexes are required in the continuous release of neurotransmitters. The exocytosis of presynaptic terminals requires a tightly coordinated membrane fusion mechanism, whose core components are SNARE-complex and Sec1/Munc18-like proteins. Numerous studies have shown that deficiency of
SNARE protein function and distribution is associated with various neurodegenerative diseases (61). In addition, studies have shown that the deposition of neurotoxic proteins can cause a wide range of neurodegenerative diseases and neuronal dysfunction, characterized by neuronal dysfunction and neuron loss (62). Typically, a-synuclein (a-Syn), the main component of amyloid protein in the Lewy body, is a neural protein expressed on the presynaptic membrane in monomeric form, which is implicated in Lewy body diseases such as Parkinson's disease (63). A $\beta$ is an amyloid protein involved in the pathogenesis of Alzheimer's disease, and the deposition of $A \beta$ is a necessary prerequisite for synaptic dysfunction and precipitation.

\section{Parkinson's disease}

Parkinson's disease (PD) is a neurodegenerative disease characterized by progressive loss of dopaminergic (DAergic) neurons in the nigrostriatal tract projected by the substantia nigra $(\mathrm{SN})$, resulting in decreased levels of dopamine in the striatum and deterioration of motor function in patients. It is speculated that a-Syn plays an important role in the pathogenesis of $\mathrm{PD}$, regulates vesicle exocytosis, including docking, priming and fusion, and is also involved in the assembly of the SNARE complex. With endogenous $\alpha$-Syn increasing the availability of synthetic vesicles at the synapse (64), the oligomerization $\alpha$-Syn contributes to the increased cytotoxicity, and the accumulation of $\alpha$-Syn showed the degeneration of dopaminergic neurons (65). In one experiment, VAMP2, which acts as a SNARE chaperone, bound to $\alpha$-Syn had no effect on vesicle exocytosis, while another assisted in attenuating neurotransmitter release upon restricting synaptic vesicle mobilization and recycling. Considering the uncertain function of presynaptic small protein $\alpha$ Syn as a cytosolic regulator, these two different views seem to be controversial. Shin (66) indicated that the capacity of aa-syn could decrease SNAREdependent membrane fusion by reducing the process of vesicle docking, while there was no effect on synaptic transmission in $\alpha$-Syn knockout mice (67). Based on the fact that synaptic vesicle (SV) recycling and exocytosis were attenuated upon the deposition of $\alpha$-Syn, the hypothesis that $\alpha$-Syn is a physiologic attenuator in the process of neurotransmitter release was proposed (68). However, there are also different opinions that $\alpha$-Syn accelerates vesicle docking and increases SNARE complex assembly. In addition, since the $\mathrm{N}$-terminus of $\alpha$-Syn can interact with the C-terminus of VAMP2, studies have shown that $\alpha$-Syn acts as a cross-bridge between vesicles and the plasma membrane (69). Diao et al. (64) used single-vesicle optical microscopy to detect the role of $\alpha$-Syn in vesicle clustering and found that $\alpha$-Syn could induce vesicle clustering, while PD-related point mutations disrupted vesicle clustering in lipid binding. It has 
also been verified that $\alpha$-Syn is related to vesicle priming and fusion pore expansion (70, 71). Therefore, it appears that the effect of $\alpha$-Syn on Parkinson's disease is mainly determined by the insufficient number of synaptic vesicles available for neurotransmitter release.

Molecular chaperones have multiple and interactive effects on accumulation. They have been useful targets for PD drug development for the aggregation and degradation of $\alpha$-synuclein by inhibiting its toxicity. Preclinical studies have shown that three compounds of ansamycin antibiotics are small-molecule inhibitors of Hsp90, including geldanamycin, tanespimycin and alvespimycin. These compounds can reduce the toxicity of $\alpha$ synuclein and promote its clearance (72). Nilotinib is a tyrosine kinase inhibitor for the treatment of adult leukaemia and has been proven to enhance the autophagy clearance rate of $\alpha$-synuclein in transgenic mice. In the lentiviral gene transfer model, autophagy can further increase the clearance of $\alpha$ synuclein and prevent neurodegeneration induced by $\alpha$-synuclein in mice (73). Anle138b is an oligomer regulator

[3-(1,3-benzodioxin-5-yl)-5-(3bromophenyl)- $1 \mathrm{H}$ pyrazole] that has been shown to prevent the formation and accumulation of $\alpha$ synuclein oligomers in the brain (74). In the human $\alpha$-synuclein mouse model, the compound has been indicated to delay the progression of the disease, even after the onset of disease-related motor dysfunction (75). In fact, anle138b markedly inhibited oligomeric volume accumulation, neuronal degeneration, and disease progression in prion disease mouse models and in three different PD mouse models (76).

\section{Alzheimer's disease}

Remarkably, recent clinical investigations indicate a strong correlation between intracellular $A \beta$ and the progression of AD symptoms. Synaptophysin, a marker of synaptic vesicles, has been shown to play important roles in calcium ion binding, channel formation, and endocytosis of synaptic vesicle recycling (77-79). Dementia in AD patients was significantly correlated with changes in synaptophysin in the hippocampus and prefrontal cortex (80). It has also been reported that the level of synaptophysin decreased by $25 \%$ in patients with $\mathrm{AD}$ compared with control subjects, while synaptotagmin and GAP43 levels remained unchanged in patients with mild $\mathrm{AD}$ (81). These results suggest that brain synaptic damage is an early case of AD. Moreover, SNARE complexes are substantially reduced in the postmortem brains of AD patients. Studies have shown that presynaptic dysfunction induced by intracellular $A \beta$ oligomers may be the pathophysiological origins of early $A D$, which is caused by the impairment of SNARE complex formation. In addition, it has been shown that intracellular amyloid- $\beta$ (A $\beta)$ inhibits SNARE- mediated exocytosis by impairing the formation of the SNARE complex (82). A $\beta 42$ has been reported to regulate the release of neurotransmitters by blocking the formation of synaptophysin and VAMP2, probably by competing with synaptophysin. A $\beta$ oligomers contribute to decreased neurotransmission efficiency by downregulating synapse density (83). In addition, $A \beta$ may cooperate with $\alpha$-Syn to inhibit SNARE-mediated vesicle fusion and cognitive dysfunction in APP/PS1 transgenic mice, showing disruption of the vesicle cycle (82). Furthermore, a clinical study (84) showed a significant negative correlation between the duration of dementia and the levels of VAMP2. In addition, the expression of VAMP1 in the brain may also modify the risk and pathophysiology of Alzheimer's disease (85). In the early stage of AD, the expression of SNARE complex-related proteins was upregulated before pathological $\alpha$-Syn extensively accumulated as a compensation for synaptic failure. Meanwhile, some other pathological proteins such as tau protein also contribute to the formation of SNARE complexes and the function of neurons in $\mathrm{AD}(86,87)$. Given the role of clathrin in amyloid precursor protein (APP) trafficking and sorting, clathrin dysfunction can cause the development of dementia, accompanied by neuron loss and clathrin-mediated endocytosis dysfunction. Previous studies have shown that the binding ability of APP to clathrin is decreased in neural stem cells of AD patients, which is related to the activation of Fyn Tyr kinase and the increase in APP Tyr phosphorylation (88). Thus, defects in the synaptic vesicle cycle may participate in the occurrence of $\mathrm{AD}$.

Immunotherapy stimulates the host immune system to identify and attack $A \beta$ or produce antibodies and improve the clearance rate of $A \beta$ oligomers or plaques, thus preventing plaque deposition. One of these $\beta$ vaccines is CAD106, which is currently undergoing clinical trials (89). Effective $A \beta$ antibody titres of various $\mathrm{IgG}$ subclasses have been induced, which mainly recognize the $A \beta 3$ 3-6 epitope. The sdpm 1 protein can bind to the $A \beta 40$ and $A \beta 42$ tetramers, which prevent the accumulation of the $A \beta$ amyloid protein. $A \beta 42$ immunization can clear amyloid plaques among patients with Alzheimer's disease but cannot prevent progressive neurodegeneration (90). A recent study showed that programmed death-1 (PD-1) inhibitors are FDA-approved anticancer drugs that may effectively clear $A \beta$ plaques and enhance cognitive ability in mouse models of Alzheimer's disease (91). $\mathrm{A} \beta$ can be degraded by various peptidases and proteases, collectively known as $\mathrm{A} \beta$-degrading protease (A $\beta$ DPS). Proteases that degrade $A \beta$ play a vital role in $A \beta$ degradation and may become good targets for the treatment of Alzheimer's disease (92).

Huntington's disease 
Huntington's disease (HD) is an autosomal dominant neurodegenerative disorder characterized by its main feature of progressive motor disturbances. It is caused by the accumulation of huntingtin protein (HTT) induced by CAG expansion in the gene. It has been reported that HTT over-expression can block the assembly of SNARE complexes and SNARE complex-mediated vesicle exocytosis (93). Recent studies have indicated that the insufficient release of presynaptic neurotransmitters is characterized by changes in function and distribution, accompanied by loss of SNAP-25 and rabphilin 3a, which are involved in vesicle docking and recycling (94). According to Rafael et al. (95), transgenic mice expressing a mutant form of htt (R6/1 mice) showed higher depression sensitivity under high-frequency stimulation and increased expression of synaptic vesicle proteins, such as VAMP1/2, SNAP-25, synaptobrevin $1 / 2$ and cysteine string protein-a, while no changes were detected in the size and dynamics of the recycling synaptic vesicle pool at the neuromuscular junction (96). Moreover, it has been demonstrated that $\mathrm{N}$-type voltage-gated $\mathrm{Ca} 2+$ channels (Cav2.2) decrease on the cell surface, accompanied by reduced glutamate release, which is induced by blocking the interaction between Cav2.2 and syntaxin-1A, suggesting the involvement of Cav2.2 in presynaptic neurotransmitter release (97). Therefore, it appears that synaptic dysfunction in Huntington's disease is mainly caused by the accumulation of protein HTT polymerization through affecting presynaptic vesicle exocytosis.

Reducing the level of mHTT can improve its toxicity in various models. In a transgenic Huntington's disease mouse model that expresses an inducible N-terminal fragment of mHTT, the reversibility of neuropathology and motor dysfunction was turned off (98). The reduced delivery of short hairpin RNA (shRNA), small interfering RNA (siRNA) or antisense oligonucleotides to mHTT attenuates neuropathological and disease-related phenotypes in several mouse models (99-102). CRISPR/Cas9mediated mHTT genome editing improves neurotoxicity in Huntington's disease (103). By catalysing the antisense oligonucleotide (ASO) mediated by RNase $\mathrm{H}$, it is proven that the short injection of CSF into a symptomatic HD mouse model not only delays the progression of the disease but also mediates the continuous reversal of the disease phenotype, which lasts longer than that of Huntington. The reduction in the wild huntingtin protein and the decrease in the mutant huntingtin protein all produce the same continuous disease reversal (104).

\section{Depression}

Depression is a common mental disorder and the leading cause of disability worldwide. According to the latest statistical figures from the World Health
Organization (WHO), depression affects approximately 300 million people worldwide and is extremely harmful to society. The National Institute of Mental Health (2020) estimates that multiple factors are involved in the development of depression, including genetic, biological, psychological factors and other factors.

In recent years, numerous studies have indicated the important roles of neurotransmission, synaptic structure and function, and abnormal neural circuits in the pathogenesis of depression (105-107). Although a number of hypotheses on the pathogenesis of depression have been proposed, including the 'monoamine hypothesis', the 'neuroplasticity hypothesis', and the 'glutamate and GABA imbalance hypothesis', the exact pathological mechanisms of depression have not been determined. Due to the limitation of intervention targets, there are no fast, effective and safe treatment drugs for this disease.

Antidepressants such as SSRIs or SSNIs that increase the synaptic concentrations of monoamines, particularly noradrenaline and serotonin, by various mechanisms can improve depressive symptoms (108). Additional evidence found that the antihypertensive reserpine depletes storage vesicles containing noradrenaline and other monoamines and produces an anti-depressive effect (109). These findings led to the adoption of the monoamine hypothesis for depression, implying that the underlying basis for depression is deficiency of central noradrenaline and/or serotonin, especially at the synaptic level. Furthermore, in clinical and animal models, numerous studies have found decreased levels of GABA and increased levels of glutamate in plasma, cerebrospinal fluid, and brain tissue in depressed patients $(110,111)$. In depression, short-term synapses are regulated by the interaction of neurexin/N-ethylmaleimide sensitive factor (NSF), resulting in impaired exocytosis through the failed disassembly of the SNARE complex (112). Meanwhile, it has also been proposed that NSF plays ancillary roles in regulating the spatial organization and in replenishing t-SNAREs to the active zone for subsequent vesicle priming (113). Taken together, this evidence implies that abnormal vesicle release may be closely associated with depression, but the underlying molecular mechanism remains unclear.

Studies have indicated that the reduction in vesicular exocytosis induced by fluoxetine (FLX) may be explained by reducing the protein levels of the main components of the SNARE core complex (SNAP-25 and VAMP-2). Additionally, these changes may be the consequence of calcium influx, which interrupts the activation of protein kinase $C$ (PKC). This interruption is due to the direct inhibition of presynaptic P/Q VGCCs by FLX (114). In the treatment of major depression, one of the most notable findings is that a single dose of ketamine infusion can cause a rapid and sustained 
antidepressant effect among patients with drugresistant depression. Ketamine-induced changes in glutamatergic tension in the HIPP and PFC trigger a molecular cascade associated with neural plasticity and then regulate the synthesis of synaptic proteins that are involved in synaptic formation (e.g., synapsin-I) and presynaptic release (e.g., synaptic fusion protein-I; Syx) (115-117). Indeed, the administration of acute low-dose ketamine has been demonstrated to rapidly increase synapsin-I protein levels and reduce the (2-ethylmaleimide sensitive factor attachment protein receptor) complex of SNARE (synaptosome preparations of soluble hip and/or PFC) composed of Syx (118).

\section{Schizophrenia}

Schizophrenia is a multifactorial, serious psychiatric disorder with disturbances in perception, cognition, and social function caused by the interplay between environmental and genetic factors (119). The treatment of schizophrenia is far from solved, partially due to the poor understanding of its underlying pathological mechanisms. Several hypotheses have been developed to address the dysregulations of the dopamine, glutamate and GABA pathways, which are involved in schizophrenia (120-122). The dopaminergic hypothesis posits that schizophrenia is induced by transmission in the basal ganglia. Recently, studies have shown that antipsychotics used to reduce the symptoms of schizophrenia decrease dopamine transmission by blocking dopamine D2 receptors $(123,124)$. This is consistent with evidence of an increased density of D2 receptors in schizophrenic human postmortem brains (125). The glutamate hypothesis posits that a deficit in glutamate neurotransmission underlies a substantial portion of the dysfunction seen in schizophrenia (122). There are three major types of glutamate receptors. Among them, NMDA receptors (NMDARs) play an important role in this hypothesis. It has been reported that certain drugs of abuse, such as ketamine, cause a constellation of symptoms that are seen in schizophrenia, which implies that NMDA receptors are involved in schizophrenia (126). Meanwhile, studies have shown that a decrease in NMDA receptor signalling in interneurons can induce cellular and behavioural changes in schizophrenia (122). Additionally, these two pathways involve a distinct molecular mechanism, but emerging evidence suggests alterations in neurotransmitter release in both hypotheses in schizophrenia. Much research has focused on determining whether presynaptic signalling proteins are involved in schizophrenia. Although data are sometimes inconsistent, different studies have found that abnormalities in the SNARE complex and its associated interacting proteins, key proteins responsible for vesicle release, might be involved in the pathogenesis of schizophrenia. Significantly, most of the studies have found that several protein machines involved in regulating synaptic vesicle transport in the brains of schizophrenic patients are decreased, such as synaptophysin, synapsin SNAP25 and complexin $1 / 2$ in the hippocampus (119). Altogether, these findings indicate a dysfunctional presynaptic vesicle release implicated in schizophrenia.

The effects of antipsychotics on trap protein levels and protein-protein interactions in the rat striatum are different from the effects in schizophrenia. For rats, haloperidol increases the levels of all three proteins in the striatum, while clozapine increases the levels of vamp. These results are consistent with previous reports in other rat brain regions $(127,128)$ and with ultrastructural studies of the effects of haloperidol on the striatum (129-131). Morphine has been reported to reduce the formation of the SNARE complex (132). Notably, in the experimental system, synthetic peptides formed by a modified SNARE complex markedly protected hippocampal neurons against glutamate-mediated neurotoxicity (133). Because of the inhibitory activity of SNARE complex formation and neuronal exocytosis, these synthetic peptides are potential drug development candidates for the treatment of neurological disorders.

\section{CONCLUSION}

Numerous studies have shown that presynaptic vesicle-mediated neurotransmitter and neuropeptide release is crucial in neurotransmission, synapse formation and remodelling, as well as the establishment of neural circuits, to maintain the normal function in the brain. Dysfunctional vesicle release in various neurotransmitter systems is closely associated with a variety of neuropsychiatric diseases, particularly depression (134), but its molecular mechanism remains unclear.

With the further understanding of the synaptic vesicle cycle, single-molecule smFRET imaging technology will be needed to monitor the interaction between proteins in real time to explore SNAREmediated membrane fusion $(118,135-137)$. It is expected that the precise role of related proteins in neurotransmitter release will be further expanded with the application of advanced bioanalytical techniques. Therefore, further investigations of the mechanisms of protein machinery components that regulate the transport and release of vesicles are warranted to elucidate their roles in the physiological and pathological processes of the development of neurological disorders. This will contribute to the investigation of new treatment targets for neurological disorders.

AUTHOR CONTRIBUTIONS. L.J.Z conceived the project; C.C. wrote the manuscript; L.J.Z. drafted and revised the manuscript; C.C.Z revised the 
manuscript. All authors reviewed and approved the final version of the manuscript.

ACKNOWLEDGEMENTS. This work was supported by the National Natural Science Foundation of China (31671107 and 31970951), and the Natural Science Foundation of Jiangsu Province (BK20170021).

CONFLICT OF INTEREST. Authors declared no conflict of interest.

\section{REFERENCES}

1. Pieribone VA, Shupliakov O, Brodin L, Hilfiker-Rothenfluh S, Czernik AJ, Greengard P. Distinct pools of synaptic vesicles in neurotransmitter release. Nature, 375(6531):493-7, 1995.

2. Rizzoli SO, Betz WJ. Synaptic vesicle pools. Nat Rev Neurosci, 6(1):57-69, 2005.

3. Cousin MA. A (free) radical approach reveals the physiological function of different synaptic vesicle pools. J Physiol, 595(4):1005-6, 2017.

4. Wesseling JF. Considerations for Measuring Activity-Dependence of Recruitment of Synaptic Vesicles to the Readily Releasable Pool. Front Synaptic Neurosci, 11:32, 2019.

5. Tarnow E. Short term memory may be the depletion of the readily releasable pool of presynaptic neurotransmitter vesicles of a metastable long term memory trace pattern. Cogn Neurodyn, 3(3):263-9, 2009.

6. Rizzoli SO, Jahn RJT. Kiss-and-run, Collapse and 'Readily Retrievable' Vesicles. 8(9):1137-44, 2007.

7. Pyle JL, Kavalali ET, Piedrasrenteria ES, Tsien RWJN. Rapid Reuse of Readily Releasable Pool Vesicles at Hippocampal Synapses. 28(1):221-31, 2000.

8. Li Z, Burrone J, Tyler WJ, Hartman KN, Albeanu DF, Murthy VN. Synaptic vesicle recycling studied in transgenic mice expressing synaptopHluorin. Proc Natl Acad Sci U S A, 102(17):6131-6, 2005.

9. Denker A, Krohnert K, Buckers J, Neher E, Rizzoli SO. The reserve pool of synaptic vesicles acts as a buffer for proteins involved in synaptic vesicle recycling. Proc Natl Acad Sci U S A, 108(41):17183-8, 2011.

10. Piccoli G, Condliffe SB, Bauer M, Giesert F, Boldt K, De Astis S, et al. LRRK2 controls synaptic vesicle storage and mobilization within the recycling pool. J Neurosci, 31(6):2225-37, 2011.

11. Pyott SJ, Rosenmund CJTJoP. The effects of temperature on vesicular supply and release in autaptic cultures of rat and mouse hippocampal neurons. 539(2):523-35, 2002.

12. Stevens CF, Sullivan JM. Regulation of the readily releasable vesicle pool by protein kinase C. Neuron, 21(4):885-93, 1998.

13. Jung J, Loy K, Schilling E, Rother M, Brauner JM, Huth T, et al. The Antidepressant Fluoxetine Mobilizes Vesicles to the Recycling Pool of Rat Hippocampal Synapses During High Activity. 49(2):916-30, 2014.

14. Jung J, Loy K, Schilling EM, Rother M, Brauner JM, Huth T, et al. Erratum to: The Antidepressant Fluoxetine Mobilizes Vesicles to the Recycling Pool of Rat Hippocampal Synapses During High Activity. Mol Neurobiol, 54(4):3117, 2017.

15. Fernandezalfonso T, Ryan TAJBCB. A heterogeneous "resting" pool of synaptic vesicles that is dynamically interchanged across boutons in mammalian CNS synapses. 36(1):87-100, 2008.

16. Vasileva M, Horstmann H, Geumann C, Gitler D, Kuner T. Synapsin-dependent reserve pool of synaptic vesicles supports replenishment of the readily releasable pool under intense synaptic transmission. Eur J Neurosci, 36(8):3005-20, 2012.

17. Schroeter A, Wen S, Molders A, Erlenhardt N, Stein V, Klocker N. Depletion of the AMPAR reserve pool impairs synaptic plasticity in a model of hepatic encephalopathy. Mol Cell Neurosci, 68:331-9, 2015.

18. Kim SH, Ryan TAJN. CDK5 serves as a major control point in neurotransmitter release. 67(5):797-809, 2010.

19. Yu H, Shen C, Liu Y, Menasche BL, Ouyang $\mathrm{Y}$, Stowell MHB, et al. SNARE zippering requires activation by SNARE-like peptides in Sec1/Munc18 proteins. Proc Natl Acad Sci U S A, 115(36):E8421-E9, 2018.

20. Japel M, Gerth F, Sakaba T, Bacetic J, Yao L, Koo SJ, et al. Intersectin-Mediated Clearance of SNARE Complexes Is Required for Fast Neurotransmission. Cell Rep, 30(2):409-20 e6, 2020.

21. Abbineni PS, Coorssen JR. Sphingolipids modulate docking, $\mathrm{Ca}(2+)$ sensitivity and membrane fusion of native cortical vesicles. Int J Biochem Cell Biol, 104:43-54, 2018.

22. Wang SSH, Held RG, Wong MY, Liu C, Karakhanyan A, Kaeser PS. Fusion Competent Synaptic Vesicles Persist upon Active Zone Disruption and Loss of Vesicle Docking. Neuron, 91(4):777-91, 2016.

23. Han GA, Malintan NT, Saw NM, Li L, Han L, Meunier FA, et al. Munc18-1 domain-1 controls vesicle docking and secretion by interacting with syntaxin-1 and chaperoning it to the plasma membrane. Mol Biol Cell, 22(21):4134-49, 2011.

24. Magdziarek M, Bolembach AA, Stepien KP, 
Quade B, Liu X, Rizo J. Re-examining how Munc13-1 facilitates opening of syntaxin-1. Protein Sci, 2020.

25. Jiao J, He M, Port SA, Baker RW, Xu Y, Qu H, et al. Munc18-1 catalyzes neuronal SNARE assembly by templating SNARE association. Elife, 7, 2018.

26. Lakomek NA, Yavuz H, Jahn R, Perez-Lara A. Structural dynamics and transient lipid binding of synaptobrevin-2 tune SNARE assembly and membrane fusion. Proc Natl Acad Sci U S A, 116(18):8699-708, 2019.

27. Wang C, Tu J, Zhang S, Cai B, Liu Z, Hou S, et al. Different regions of synaptic vesicle membrane regulate VAMP2 conformation for the SNARE assembly. Nat Commun, 11(1):1531, 2020.

28. Yoon TY, Munson M. SNARE complex assembly and disassembly. Curr Biol, 28(8):R397-R401, 2018.

29. Melia TJ, Weber T, McNew JA, Fisher LE, Johnston RJ, Parlati F, et al. Regulation of membrane fusion by the membrane-proximal coil of the t-SNARE during zippering of SNAREpins. J Cell Biol, 158(5):929-40, 2002.

30. Yang Y, Shin JY, Oh JM, Jung CH, Hwang Y, Kim S, et al. Dissection of SNARE-driven membrane fusion and neuroexocytosis by wedging small hydrophobic molecules into the SNARE zipper. Proc Natl Acad Sci U S A, 107(51):22145-50, 2010.

31. Grushin K, Wang J, Coleman J, Rothman JE, Sindelar CV, Krishnakumar SS. Structural basis for the clamping and $\mathrm{Ca}(2+)$ activation of SNARE-mediated fusion by synaptotagmin. Nat Commun, 10(1):2413, 2019.

32. He R, Zhang J, Yu Y, Jizi L, Wang W, Li M. New Insights Into Interactions of Presynaptic Calcium Channel Subtypes and SNARE Proteins in Neurotransmitter Release. Front Mol Neurosci, 11:213, 2018.

33. Sharma S, Lindau M. Molecular mechanism of fusion pore formation driven by the neuronal SNARE complex. Proc Natl Acad Sci U S A, 115(50):12751-6, 2018.

34. Song H, Wickner W. Tethering guides fusioncompetent trans-SNARE assembly. Proc Natl Acad Sci U S A, 116(28):13952-7, 2019.

35. Vivona S, Cipriano DJ, O'Leary S, Li YH, Fenn TD, Brunger AT. Disassembly of all SNARE complexes by N-ethylmaleimidesensitive factor (NSF) is initiated by a conserved 1:1 interaction between alphasoluble NSF attachment protein (SNAP) and SNARE complex. J Biol Chem, 288(34):24984-91, 2013.

36. HT M, biology BEJNrMc. Molecular mechanism and physiological functions of clathrin-mediated endocytosis. 12(8):517-33, 2011.
37. Gulbranson DR, Crisman L, Lee M, Ouyang Y, Menasche BL, Demmitt BA, et al. AAGAB Controls AP2 Adaptor Assembly in ClathrinMediated Endocytosis. Dev Cell, 50(4):43646 e $5,2019$.

38. Manna PT, Gadelha C, Puttick AE, Field MC. ENTH and ANTH domain proteins participate in AP2-independent clathrin-mediated endocytosis. J Cell Sci, 128(11):2130-42, 2015.

39. Kaksonen M, Roux A. Mechanisms of clathrin-mediated endocytosis. Nat Rev Mol Cell Biol, 19(5):313-26, 2018.

40. Baschieri F, Porshneva K, Montagnac G. Frustrated clathrin-mediated endocytosis causes and possible functions. J Cell Sci, 133(11), 2020.

41. Fesce R, Grohovaz F, Valtorta F, Meldolesi J. Neurotransmitter release: fusion or 'kiss-andrun'? Trends in Cell Biology, 4(1):1-4, 1994.

42. Ferrarelli LK. Kiss-and-run or a full commitment? Science, 356(6344):1243-5, 2017.

43. Wen X, Saltzgaber GW, Thoreson WB. Kissand-Run Is a Significant Contributor to Synaptic Exocytosis and Endocytosis in Photoreceptors. Front Cell Neurosci, 11:286, 2017.

44. Alabi AA, Tsien RW. Perspectives on kissand-run: role in exocytosis, endocytosis, and neurotransmission. Annu Rev Physiol, 75:393-422, 2013

45. Jackson J, Papadopulos A, Meunier F, McCluskey A, Robinson P, Keating D. Small molecules demonstrate the role of dynamin as a bi-directional regulator of the exocytosis fusion pore and vesicle release. Molecular psychiatry, 20, 2015.

46. Watanabe S, Rost BR, Camachoperez M, Davis MW, Sohlkielczynski B, Rosenmund C, et al. Ultrafast endocytosis at mouse hippocampal synapses. 504(7479):242-7, 2013.

47. Watanabe S, Liu Q, Davis MW, Hollopeter G, Thomas N, Jorgensen NB, et al. Ultrafast endocytosis at Caenorhabditis elegans neuromuscular junctions. 2(2), 2013.

48. Soykan T, Kaempf N, Sakaba T, Vollweiter D, Goerdeler F, Puchkov D, et al. Synaptic Vesicle Endocytosis Occurs on Multiple Timescales and Is Mediated by ForminDependent Actin Assembly. Neuron, 93(4):854-66 e4, 2017.

49. Watanabe S, Rost BR, Camacho-Pérez M, Davis MW, Söhl-Kielczynski B, Rosenmund $\mathrm{C}$, et al. Ultrafast endocytosis at mouse hippocampal synapses. Nature, 504(7479):242-7, 2013.

50. Mahapatra S, Fan F, Lou X. Tissue-specific dynamin-1 deletion at the calyx of Held 
decreases short-term depression through a mechanism distinct from vesicle resupply. Proc Natl Acad Sci U S A, 113(22):E3150-8, 2016.

51. Watanabe S, Mamer LE, Raychaudhuri S, Luvsanjav D, Eisen J, Trimbuch T, et al. Synaptojanin and Endophilin Mediate Neck Formation during Ultrafast Endocytosis. Neuron, 98(6):1184-97 e6, 2018.

52. Yu SC, Janosi B, Liewald JF, Wabnig S, Gottschalk A. Endophilin A and B Join Forces With Clathrin to Mediate Synaptic Vesicle Recycling in Caenorhabditis elegans. Front Mol Neurosci, 11:196, 2018.

53. Cheung G, Jupp OJ, Cousin MA. Activitydependent bulk endocytosis and clathrindependent endocytosis replenish specific synaptic vesicle pools in central nerve terminals. J Neurosci, 30(24):8151-61, 2010.

54. G C, neurochemistry CMJJo. Synaptic vesicle generation from activity-dependent bulk endosomes requires a dephosphorylationdependent dynamin-syndapin interaction. 151(5):570-83, 2019.

55. Morton A, Marland JR, Cousin MA. Synaptic vesicle exocytosis and increased cytosolic calcium are both necessary but not sufficient for activity-dependent bulk endocytosis. J Neurochem, 134(3):405-15, 2015.

56. Cheung G, Cousin MA. Synaptic Vesicle Generation from Activity-Dependent Bulk Endosomes Requires Calcium and Calcineurin. The Journal of Neuroscience, 33(8):3370, 2013.

57. Kokotos AC, Low DWJTJoN. Myosin II and Dynamin Control Actin Rings to Mediate Fission during Activity-Dependent Bulk Endocytosis. 35(23):8687-8, 2015.

58. Nicholson-Fish JC, Kokotos AC, Gillingwater $\mathrm{TH}$, Smillie KJ, Cousin MA. VAMP4 Is an Essential Cargo Molecule for ActivityDependent Bulk Endocytosis. Neuron, 88(5):973-84, 2015.

59. Kokotos AC, Peltier J, Davenport EC, Trost M, Cousin MA. Activity-dependent bulk endocytosis proteome reveals a key presynaptic role for the monomeric GTPase Rab11. Proc Natl Acad Sci U S A, 115(43):E10177-E86, 2018.

60. Silm K, Yang J, Marcott PF, Asensio CS, Eriksen J, Guthrie DA, et al. Synaptic Vesicle Recycling Pathway Determines Neurotransmitter Content and Release Properties. Neuron, 102(4):786-800 e5, 2019.

61. Hou C, Wang Y, Liu J, Wang C, Long J. Neurodegenerative Disease Related Proteins Have Negative Effects on SNARE-Mediated Membrane Fusion in Pathological Confirmation. Front Mol Neurosci, 10:66, 2017.
62. Lee YI, Kim YG, Pyeon HJ, Ahn JC, Logan S, Orock A, et al. Dysregulation of the SNAREbinding protein Munc18-1 impairs BDNF secretion and synaptic neurotransmission: a novel interventional target to protect the aging brain. Geroscience, 41(2):109-23, 2019.

63. Mukaetova-Ladinska EB, Xuereb JH, GarciaSierra F, Hurt J, Gertz HJ, Hills R, et al. Lewy body variant of Alzheimer's disease: selective neocortical loss of t-SNARE proteins and loss of MAP2 and alpha-synuclein in medial temporal lobe. ScientificWorldJournal, 9:1463-75, 2009.

64. Diao J, Burre J, Vivona S, Cipriano DJ, Sharma M, Kyoung M, et al. Native alphasynuclein induces clustering of synapticvesicle mimics via binding to phospholipids and synaptobrevin-2/VAMP2. Elife, 2:e00592, 2013.

65. Hogen T, Levin J, Schmidt F, Caruana M, Vassallo N, Kretzschmar $\mathrm{H}$, et al. Two different binding modes of alpha-synuclein to lipid vesicles depending on its aggregation state. Biophys J, 102(7):1646-55, 2012.

66. Lai Y, Kim S, Varkey J, Lou X, Song JK, Diao $\mathrm{J}$, et al. Nonaggregated alpha-synuclein influences SNARE-dependent vesicle docking via membrane binding. Biochemistry, 53(24):3889-96, 2014.

67. Nemani VM, Lu W, Berge V, Nakamura K, Onoa B, Lee MK, et al. Increased expression of alpha-synuclein reduces neurotransmitter release by inhibiting synaptic vesicle reclustering after endocytosis. Neuron, 65(1):66-79, 2010.

68. Sun J, Wang L, Bao H, Premi S, Das U, Chapman ER, et al. Functional cooperation of alpha-synuclein and VAMP2 in synaptic vesicle recycling. Proc Natl Acad Sci U S A, 116(23):11113-5, 2019.

69. Huang M, Wang B, Li X, Fu C, Wang C, Kang $\mathrm{X}$. alpha-Synuclein: A Multifunctional Player in Exocytosis, Endocytosis, and Vesicle Recycling. Front Neurosci, 13:28, 2019.

70. Wang C, Ma Z, Yan DY, Liu C, Deng Y, Liu $\mathrm{W}$, et al. Alpha-Synuclein and Calpains Disrupt SNARE-Mediated Synaptic Vesicle Fusion During Manganese Exposure in SHSY5Y Cells. Cells, 7(12), 2018.

71. Logan T, Bendor J, Toupin C, Thorn K, Edwards RH. alpha-Synuclein promotes dilation of the exocytotic fusion pore. Nat Neurosci, 20(5):681-9, 2017.

72. Kim YS, Alarcon SV, Lee S, Lee MJ, Giaccone G, Neckers L, et al. Update on Hsp90 inhibitors in clinical trial. Curr Top Med Chem, 9(15):1479-92, 2009.

73. Hebron ML, Lonskaya I, Moussa CE. Nilotinib reverses loss of dopamine neurons and improves motor behavior via autophagic 
degradation of alpha-synuclein in Parkinson's disease models. Hum Mol Genet, 22(16):3315-28, 2013.

74. Dehay B, Decressac M, Bourdenx $M$, Guadagnino I, Fernagut PO, Tamburrino A, et al. Targeting alpha-synuclein: Therapeutic options. Mov Disord, 31(6):882-8, 2016.

75. Levin J, Schmidt F, Boehm C, Prix C, Botzel $\mathrm{K}$, Ryazanov $\mathrm{S}$, et al. The oligomer modulator anle138b inhibits disease progression in a Parkinson mouse model even with treatment started after disease onset. Acta Neuropathol, 127(5):779-80, 2014.

76. Wagner J, Ryazanov S, Leonov A, Levin J, Shi S, Schmidt F, et al. Anle138b: a novel oligomer modulator for disease-modifying therapy of neurodegenerative diseases such as prion and Parkinson's disease. Acta Neuropathol, 125(6):795-813, 2013.

77. Kokotos AC, Harper CB, Marland JRK, Smillie KJ, Cousin MA, Gordon SL. Synaptophysin sustains presynaptic performance by preserving vesicular synaptobrevin-II levels. J Neurochem, 151(1):28-37, 2019.

78. Leimer U, Franke WW, Leube RE. Synthesis of the mammalian synaptic vesicle protein synaptophysin in insect cells: a model for vesicle biogenesis. Exp Cell Res, 224(1):8895, 1996.

79. Rehm H, Wiedenmann B, Betz H. Molecular characterization of synaptophysin, a major calcium-binding protein of the synaptic vesicle membrane. EMBO J, 5(3):535-41, 1986.

80. Glantz LA, Gilmore JH, Hamer RM, Lieberman JA, Jarskog LFJN. Synaptophysin and postsynaptic density protein 95 in the human prefrontal cortex from mid-gestation into early adulthood. 149(3):582-91, 2007.

81. Masliah E, Mallory M, Alford M, Deteresa R, Hansen LA, Mckeel DW, et al. Altered expression of synaptic proteins occurs early during progression of Alzheimer's disease. 56(1):127-9, 2001.

82. Yang Y, Kim J, Kim HY, Ryoo N, Lee S, Kim Y, et al. Amyloid-beta Oligomers May Impair SNARE-Mediated Exocytosis by Direct Binding to Syntaxin 1a. Cell Rep, 12(8):124451, 2015.

83. Lacor PN, Buniel MC, Furlow PW, Clemente AS, Velasco PT, Wood M, et al. Abeta oligomer-induced aberrations in synapse composition, shape, and density provide a molecular basis for loss of connectivity in Alzheimer's disease. J Neurosci, 27(4):796807, 2007.

84. Vallortigara J, Whitfield D, Quelch W, Alghamdi A, Howlett D, Hortobagyi T, et al. Decreased Levels of VAMP2 and Monomeric
Alpha-Synuclein Correlate with Duration of Dementia. J Alzheimers Dis, 50(1):101-10, 2016.

85. Sevlever D, Zou F, Ma L, Carrasquillo S, Crump MG, Culley OJ, et al. Geneticallycontrolled Vesicle-Associated Membrane Protein 1 expression may contribute to Alzheimer's pathophysiology and susceptibility. Mol Neurodegener, 10:18, 2015.

86. Moreau K, Fleming A, Imarisio S, Lopez Ramirez A, Mercer JL, Jimenez-Sanchez M, et al. PICALM modulates autophagy activity and tau accumulation. Nat Commun, 5:4998, 2014.

87. Mcinnes J, Wierda K, Snellinx A, Bounti L, Wang Y, Stancu I, et al. Synaptogyrin-3 Mediates Presynaptic Dysfunction Induced by Tau. 97(4):823-35, 2018.

88. Poulsen ET, Iannuzzi F, Rasmussen HF, Maier TJ, Enghild JJ, Jorgensen AL, et al. An Aberrant Phosphorylation of Amyloid Precursor Protein Tyrosine Regulates Its Trafficking and the Binding to the Clathrin Endocytic Complex in Neural Stem Cells of Alzheimer's Disease Patients. Front Mol Neurosci, 10:59, 2017

89. Fu Z, Aucoin D, Ahmed M, Ziliox M, Van Nostrand WE, Smith SO. Capping of abeta42 oligomers by small molecule inhibitors. Biochemistry, 53(50):7893-903, 2014.

90. Mueller-Steiner S, Zhou Y, Arai H, Roberson ED, Sun B, Chen J, et al. Antiamyloidogenic and neuroprotective functions of cathepsin B: implications for Alzheimer's disease. Neuron, 51(6):703-14, 2006.

91. Fluhrer R, Multhaup G, Schlicksupp A, Okochi M, Takeda M, Lammich S, et al. Identification of a beta-secretase activity, which truncates amyloid beta-peptide after its presenilin-dependent generation. J Biol Chem, 278(8):5531-8, 2003.

92. Huang SM, Mouri A, Kokubo H, Nakajima R, Suemoto T, Higuchi M, et al. Neprilysinsensitive synapse-associated amyloid-beta peptide oligomers impair neuronal plasticity and cognitive function. $\mathrm{J}$ Biol Chem, 281(26):17941-51, 2006.

93. King AC, Wood TE, Rodriquez E, Parpura V, Gray M. Differential effects of SNAREdependent gliotransmission on behavioral phenotypes in a mouse model of Huntington's disease. Exp Neurol:113358, 2020.

94. Smith R, Klein P, Koc-Schmitz Y, Waldvogel HJ, Faull RL, Brundin P, et al. Loss of SNAP25 and rabphilin $3 \mathrm{a}$ in sensory-motor cortex in Huntington's disease. J Neurochem, 103(1):115-23, 2007.

95. Rozas JL, Gomez-Sanchez L, Tomas-Zapico C, Lucas JJ, Fernandez-Chacon R. Increased neurotransmitter release at the neuromuscular 
junction in a mouse model of polyglutamine disease. J Neurosci, 31(3):1106-13, 2011.

96. Doria JG, De Souza JM, Silva FR, Olmo IG, Carvalho TG, Alvessilva J, et al. The mGluR5 positive allosteric modulator VU0409551 improves synaptic plasticity and memory of a mouse model of Huntington's disease. 147(2):222-39, 2018.

97. Silva FR, Miranda AS, Santos RPM, Olmo IG, Zamponi GW, Dobransky T, et al. N-type $\mathrm{Ca}(2+)$ channels are affected by full-length mutant huntingtin expression in a mouse model of Huntington's disease. Neurobiol Aging, 55:1-10, 2017.

98. Yamamoto A, Lucas JJ, Hen R. Reversal of neuropathology and motor dysfunction in a conditional model of Huntington's disease. Cell, 101(1):57-66, 2000.

99. Harper SQ, Staber PD, He X, Eliason SL, Martins IH, Mao Q, et al. RNA interference improves motor and neuropathological abnormalities in a Huntington's disease mouse model. Proc Natl Acad Sci U S A, 102(16):5820-5, 2005.

100. Rodriguez-Lebron E, Denovan-Wright EM, Nash K, Lewin AS, Mandel RJ. Intrastriatal rAAV-mediated delivery of anti-huntingtin shRNAs induces partial reversal of disease progression in R6/1 Huntington's disease transgenic mice. Mol Ther, 12(4):618-33, 2005.

101. DiFiglia M, Sena-Esteves M, Chase K, Sapp E, Pfister E, Sass M, et al. Therapeutic silencing of mutant huntingtin with siRNA attenuates striatal and cortical neuropathology and behavioral deficits. Proc Natl Acad Sci U S A, 104(43):17204-9, 2007.

102. Kordasiewicz HB, Stanek LM, Wancewicz EV, Mazur C, McAlonis MM, Pytel KA, et al. Sustained therapeutic reversal of Huntington's disease by transient repression of huntingtin synthesis. Neuron, 74(6):1031-44, 2012.

103. Yang S, Chang R, Yang H, Zhao T, Hong Y, Kong HE, et al. CRISPR/Cas9-mediated gene editing ameliorates neurotoxicity in mouse model of Huntington's disease. J Clin Invest, 127(7):2719-24, 2017.

104. Stanford W, Galvin J, Rooholamini M. Effects of awake tidal breathing, swallowing, nasal breathing, oral breathing and the Muller and Valsalva maneuvers on the dimensions of the upper airway. Evaluation by ultrafast computerized tomography. Chest, 94(1):14954, 1988.

105. Krishnan V, Nestler EJ. The molecular neurobiology of depression. Nature, 455(7215):894-902, 2008.

106. Duman RS, Aghajanian GK. Synaptic dysfunction in depression: potential therapeutic targets. Science, 338(6103):68-72,

\section{2.}

107. Ressler KJ, Mayberg HS. Targeting abnormal neural circuits in mood and anxiety disorders: from the laboratory to the clinic. Nat Neurosci, 10(9):1116-24, 2007.

108. Racagni G, Popoli M. Cellular and molecular mechanisms in the long-term action of antidepressants. Dialogues Clin Neurosci, 10(4):385-400, 2008.

109. Heninger GR, Delgado PL, Charney DS. The revised monoamine theory of depression: a modulatory role for monoamines, based on new findings from monoamine depletion experiments in humans. Pharmacopsychiatry, 29(1):2-11, 1996.

110. Brambilla P, Perez J, Barale F, Schettini G, Soares JC. GABAergic dysfunction in mood disorders. Mol Psychiatry, 8(8):721-37, 15, 2003.

111. Sanacora G, Zarate CA, Krystal JH, Manji HK Targeting the glutamatergic system to develop novel, improved therapeutics for mood disorders. Nat Rev Drug Discov, 7(5):426-37, 2008.

112. Li T, Tian Y, Li Q, Chen H, Lv H, Xie W, et al. The Neurexin/N-Ethylmaleimide-sensitive Factor (NSF) Interaction Regulates Short Term Synaptic Depression. J Biol Chem, 290(29):17656-67, 2015.

113. Kawasaki F, Ordway RW. Molecular mechanisms determining conserved properties of short-term synaptic depression revealed in NSF and SNAP-25 conditional mutants. Proc Natl Acad Sci U S A, 106(34):14658-63, 2009.

114. Jenike MA. The use of monoamine oxidase inhibitors in the treatment of elderly, depressed patients. J Am Geriatr Soc, 32(8):571-5, 1984.

115. Abdallah CG, Sanacora G, Duman RS, Krystal JH. Ketamine and rapid-acting antidepressants: a window into a new neurobiology for mood disorder therapeutics. Annu Rev Med, 66:509-23, 2015.

116. Li N, Lee B, Liu RJ, Banasr M, Dwyer JM, Iwata $\mathrm{M}$, et al. mTOR-dependent synapse formation underlies the rapid antidepressant effects of NMDA antagonists. Science, 329(5994):959-64, 2010.

117. Muller HK, Wegener G, Liebenberg N, Zarate CA, Jr., Popoli M, Elfving B. Ketamine regulates the presynaptic release machinery in the hippocampus. J Psychiatr Res, 47(7):8929, 2013.

118. Zhao B, Watanabe G, Morten MJ, Reid DA, Rothenberg E, Lieber MR. The essential elements for the noncovalent association of two DNA ends during NHEJ synapsis. Nat Commun, 10(1):3588, 2019.

119. Egbujo CN, Sinclair D, Hahn CG. 
Dysregulations of Synaptic Vesicle Trafficking in Schizophrenia. Curr Psychiatry Rep, 18(8):77, 2016.

120. Antonucci F, Corradini I, Morini R, Fossati G, Menna E, Pozzi D, et al. Reduced SNAP-25 alters short-term plasticity at developing glutamatergic synapses. Embo Reports, 14(7):645-51, 2013.

121. Anzalone A, Lizardi-Ortiz JE, Ramos M, De Mei C, Hopf FW, Iaccarino C, et al. Dual control of dopamine synthesis and release by presynaptic and postsynaptic dopamine D2 receptors. J Neurosci, 32(26):9023-34, 2012.

122. Gordon JA. Testing the glutamate hypothesis of schizophrenia. Nat Neurosci, 13(1):2-4, 2010.

123. Stahl SM. Beyond the dopamine hypothesis of schizophrenia to three neural networks of psychosis: dopamine, serotonin, and glutamate. CNS Spectr, 23(3):187-91, 2018.

124. Szczypinski JJ, Gola M. Dopamine dysregulation hypothesis: the common basis for motivational anhedonia in major depressive disorder and schizophrenia? Rev Neurosci, 29(7):727-44, 2018.

125. Zakzanis KK, Hansen KT. Dopamine D2 densities and the schizophrenic brain. Schizophrenia research, 32(3):201-6, 1998.

126. Krystal JH, Anand A, Moghaddam B. Effects of NMDA receptor antagonists: implications for the pathophysiology of schizophrenia. Arch Gen Psychiatry, 59(7):663-4, 2002.

127. Barr AM, Young CE, Phillips AG, Honer WG. Selective effects of typical antipsychotic drugs on SNAP-25 and synaptophysin in the hippocampal trisynaptic pathway. Int $\mathrm{J}$ Neuropsychopharmacol, 9(4):457-63, 2006.

128. Sawada K, Young CE, Barr AM, Longworth K, Takahashi S, Arango V, et al. Altered immunoreactivity of complexin protein in prefrontal cortex in severe mental illness. Mol Psychiatry, 7(5):484-92, 2002.

129. Benes FM, Paskevich PA, Davidson J, Domesick VB. The effects of haloperidol on synaptic patterns in the rat striatum. Brain Res, 329(1-2):265-73, 1985.

130. Kerns JM, Sierens DK, Kao LC, Klawans HL, Carvey PM. Synaptic plasticity in the rat striatum following chronic haloperidol treatment. Clin Neuropharmacol, 15(6):488500, 1992.

131. Uranova NA, Orlovskaya DD, Apel K, Klintsova AJ, Haselhorst U, Schenk H. Morphometric study of synaptic patterns in the rat caudate nucleus and hippocampus under haloperidol treatment. Synapse, 7(4):253-9, 1991.

132. Xu NJ, Yu YX, Zhu JM, Liu H, Shen L, Zeng $\mathrm{R}$, et al. Inhibition of SNAP-25 phosphorylation at Ser187 is involved in chronic morphine-induced down-regulation of SNARE complex formation. J Biol Chem, 279(39):40601-8, 2004.

133. Blanes-Mira C, Merino JM, Valera E, Fernandez-Ballester G, Gutierrez LM, Viniegra $\mathrm{S}$, et al. Small peptides patterned after the N-terminus domain of SNAP25 inhibit SNARE complex assembly and regulated exocytosis. J Neurochem, 88(1):124-35, 2004.

134. Shabel SJ, Proulx CD, Piriz J, Malinow R. Mood regulation. GABA/glutamate corelease controls habenula output and is modified by antidepressant treatment. Science, 345(6203):1494-8, 2014.

135. Choi UB, Zhao M, White KI, Pfuetzner RA, Esquivies L, Zhou Q, et al. NSF-mediated disassembly of on- and off-pathway SNARE complexes and inhibition by complexin. Elife, 7, 2018.

136. Hiersemenzel K, Brown ER, Duncan RR. Imaging large cohorts of single ion channels and their activity. Front Endocrinol (Lausanne), 4:114, 2013.

137. Hu Y, Tian Z, Diao J. Single-Molecule Fluorescence Measurement of SNAREMediated Vesicle Fusion. Methods Mol Biol, 1860:335-44, 2019. 